\title{
Creativity and communication in the Visual Arts
}

\author{
Gabriela BENESCU ${ }^{1}$
}

\begin{abstract}
Communication through art involves knowing the specific means of creative expression in the visual arts, understanding the dynamics of the evolution of visual language and its use. The quality, the value of a work of art is given by the harmonious cooperation of the modalities of plastic expression with the help of which the idea, the subject is transposed and of the openness that it has towards the possibilities of communication. For optimal reception of the artistic product, educational support in the field is very important. At the same time, visual art education helps the individual to develop sensory and cognitive skills, facilitating communication with others, but also with him, which will lead to a deeper understanding of reality. All these particularities, used and exploited by the visual artists and, implicitly, transmitted to others lead to the outline of moral and spiritual values, to the completion of human evolution.
\end{abstract}

Key-words: creativity, communication, composition, education

\section{Introduction}

Visual arts are an exciting opportunity to express thoughts in images. Most often through the image, feelings are better communicated than words, because the graphic symbols and images carry messages, ideas and actions, being much easier to express. In addition to the aesthetic and knowledge role, visual education plays an important role in communication. The activity process is oriented to analysis, observation, introspection, creative expression but also to the discovery and understanding of language, content in different cultural contexts of space and time. The elements of plastic language being common in different cultures facilitate nonverbal communication at the global level.

\section{Objectives}

Visual expression can be a form of balance between the inner and outer experiences; it can lead to a better self-knowledge. Educational activities are also an alternative way of knowing from the perspective of the individual potential and

${ }^{1}$ National University of Arts “G. Enescu” of lași, gabrielabenescu@yahoo.com 
the cultural level, to identify the psycho-individual and age particularities. Throughout life, under the influence of socio-cultural factors and due to the interaction with everyday experiences, creativity can develop progressively, concurrently with the other psychic processes. The social and family context, the education, the geographical space put their mark in the stimulation of the intellectual, visual and spiritual curiosity, in the process of affective and cognitive development. Visual education proposes the development of skills, attitudes in the field of culture, creative communication and cooperation, promotion and preservation of cultural values. The evolution of the visual representations, with the corresponding symbolic loads, offers a significant thematic reflection while also constituting a complex aesthetic experience. The human being has always organized its existence as a microcosm in which the forms, the signs, the symbols represent the encoding of their own experience expressed through their own language. Forms of expression become over time models of human need to reconstruct. Once released from the material side of life and discovering the aesthetic dimension, human can access moments of upliftment, of beauty and spiritual joy. The dynamics of changes in today's society require a constant reassessment of the educational role according to the cognitive experience of humanity. The quality is given by the flexibility and openness to the complex demands of contemporary education, the possibility of being in line with all the changes and transformations that have occurred in the progress of society.

\section{Specific methods and elements to visual communication - configuration and reception}

Visual arts propose models and aim to produce aesthetic values, using specific means of expression. Receiving a work of art is similar to reading a book, if the signs, the meaning of the words, the grammar are known to us, the message can be deciphered. As spoken or written language contains words, musical language is expressed through notes, and the visual arts have their own language based on signs that demand from the viewer a certain degree of visual culture and education. If we do not know these specific means of communication, we cannot understand the message of a work of art.

Time has shown that each era, each period promotes distinct evaluation criteria. In the pictorial space many of the basic compositional, chromatic principles have certain constancy. The intimate constructive system of the operating space essentially has certain compositional types that have not lost their validity in time. The current aesthetic attitudes and orientations cannot be radically detached from this operative, ordinating nucleus. The direct interaction with the authentic work of art can be an exercise in how a contemporary artist can interrogate the art of the 
masters, in order to find another possibility to put the creative approach into question. It concerns the coherence of the visual expression that allows both the deciphering of the works of the various systems and the access to a personal expression. The art aims to liberate human from limiting mimesis, proposing the conversion of known things into signs with multiple meanings. The complex process of aesthetic perception is not limited to the only satisfaction of identifying recognizable elements or narration. The art work being a space of suggestions and symbols, a set of sensory challenges, gives the contemplator the freedom of interpretation (Ailincai 1982, 15-21). Formal and chromatic components always form new relationships, influencing the perceptual, affective, emotional moment differently. Through the eyes of those who see creation, new and new projections become a continuous revelation. For example, in the work "Rotation" (Paul Klee), realized in blue and black ink, in which the composition suggests a galaxy in slow motion around active points, with rotating centrifugal pulses, the author freed himself from the figurative reports keeping possible similarities. The subject represented appears to be a flower, a woman, a cat, a man, an apple, a circle. Released by the constraint of a perfectly defined work, it allows the art consumer to create again, in a personal way, to become a co-author himself. The works of art must respond to conceptual characteristics through the ideas processed to be communicated with the necessary openness to give the viewers multiple possibilities of interpretation. Regarding this idea, Kandinsky says that a work of art: "is born secretly from the artist. Detached from it, the work acquires an autonomous life; she becomes a personality herself, an independent subject with a spiritual soul, but also possessing a real material life, existing as a being" (1994, 109). Visual work acts, participates, transmits the spiritual atmosphere and as no living being ever exhausts her creative forces. The dialogue between the work of art and the viewer occurs through the specific means of communication but also through the most sensitive ways of the soul. In general, an art work is perceived and judged in direct relation to a number of factors that define the human structure. The degree of education, culture influences the potential for understanding. The aesthetic value of a work of art is given by the harmonious collaboration of the modalities of visual expression with the help of which the idea, the subject is transposed. The realization of the perceptive act at a higher qualitative level is conditioned by certain factors, such as: the knowledge fund, the acquisition of the specific language, the previous experience, the voluntary effort respectively the active attitude. This active attitude is expressed on the one hand through a series of motor acts such as: looking from different angles and distances, touching etc. and on the other hand through a series of complex mental actions such as comparison, synthesis, abstraction, generalization, etc. Thus, being reflected more deeply, the images of reality lead to a variety of modes of representation and implicit reception. Reality is revealed to us under new meanings 
and meanings, revealing it each time in new, unrepeatable forms. H. C. Gadamer, starting from the philosophical ideas of Kant who understands the creative genius as a force of nature considering him the favorite of nature, from the perspective of his own vision, explains this belief. The artist in a close relationship with nature, "is favored by it in such a way that it creates, just like her, not in a conscious adaptation to some rules, something that is also an invoice as if it were made by rules - even more: as if it were something unheard of, created by rules he had never understood before. This is art: it creates something impeccable, without producing that one by all rules. At the same time, the determination of art as a creation of genius must not under any circumstances be separated from the congeniality of the receiver. Both are a free game" (Gadamer 2000, 86). The representations that support the visual space mean the concretization of the artist's thinking, cultural richness, education, sentimental state, psychic reaction to the metamorphoses of the world. The reality perceived by human manifests itself in a differentiated way. In order to enjoy the spiritual universe of art, it is necessary to understand and deepen the fruit of knowledge, to direct emotions towards authentic aesthetic values. The painter's vision fails to achieve its goal of being optimally transmitted if the viewer does not know the signs of visual language and does not resonate with the conceptualized idea. The way of configuring the visual representations, being in a perpetual change, forces the receiver to be constantly involved in the process of information, of deciphering the specific signs.

\section{Factors of visual expressivity}

Concerns related to finding new ways to configure the image require thorough preparation through documentation, analysis and informational interpretation. Understanding the descriptive functions and then overcoming them by trying to penetrate beyond the formal appearances, the reflections on real data are reflected through materialization of multiple visual configurations. The results of the investigations then lead to mental reasonings, imaginary projects for new combinations, new substitutions, the searches continuing until the best solutions are found. "Nature does not imply an approach only with the involvement of the sentimental side, it forces us to think. We see something, but we know that what we see is only a fragment of reality. We realize that on this side and beyond that bit, the extent of space and time is infinite, the cosmic forces that generate the phenomena are powerful and obscure, we move our minds beyond what we see and what is visible around us, in the realm of dreams, memory, fantasy, forecasts, and intuition. What we see loses any interest. What we do not see, but we think it exists, imposes itself and frightens us through its infinity, awakening in us the anxiety about our own limits. This transcendental reality is the sublime" (Argan 1982, 62). 
The use of sensory knowledge eases the effort towards rational knowledge, stimulates mental activism and forms the spirit of observation. Through the exercise it is possible to expand the area of knowledge and to increase the skills in practice, experiences that in turn can be shared. Given the idea of interdisciplinarity in education, in addition to the development of the specific information area, logical associations between knowledge, concepts assimilated from different cultural areas that contribute to the sound development of cognitive-aptitude capacities are encouraged. The training of the skills of assimilation and synthesis, of the conception of the original artistic creation in resonance with the current and perspective trends of the visual arts on European and world level, is a constant in the concerns regarding the creative process. The good relationship between the elements of plastic language, the color harmonies, the rhythms and the value contrasts, are factors of the plastic expressiveness capable of determining aesthetic emotions. "Man through art, expresses himself more completely, understands himself, he is better, and the world around him becomes more accessible and intelligible." (Huyghe 1981, 13)

They become homogeneous microstructures of visual language, to produce on the viewer emotion and unity in the impression. It is important the harmonious cooperation of the various visual elements in order to acquire the sense of order and balance. The way of distribution of the different elements, their proportions regulate the inner movement of the compositional image through which the surface, lines, colors etc. I aim to adjust the relationships in the plastic space, in order to ensure its cohesion and the overall expressiveness. There are aspects found in countless works, although the problems generated by the configuration of the visual space are not always similar (Ortega y Gasset 2000, 81-86).

Respecting the generally valid principles, the configuration of the compositional space can be made by establishing a constant relation of mutual dependence between the parts and the whole, taking into account all the factors of compositional organization. The spatial density direction of the field and its expansion directions also structurally determines the composition. Relationships between points, lines, surfaces, colors, volumes, etc. they can be thought and felt based on the problems of: proportions, contrasts, rhythms, balance, unity and diversity, expressivity, harmony, meanings, etc. The configuration of the compositional space is based on certain harmony relationships between the component parts, as well as between these parts and the whole. Thus, a system of mutual relations is generated, in which the whole conditions and disposes of the parties, and the parties cooperate by revealing the whole.

\subsection{Unity and variety}

The unit resides in the co-operation of the various visual elements in order to acquire the sense of order and to achieve a harmonious balance between the different parts of 
the whole. The way of subordination of the individual parts, the whole concept is considered to be important. Each element yields part of its unit to the assembly. The composition of the different elements produces and regulates the inner movement through which the different elements of the plastic language seek to regulate the relations in order to ensure cohesion. This inner solidarity is intended to express the unity of expression which converts all the elements in the same sense and which, by changing the nature of our view, elevates the spirit and the sensitivity. There are aspects found in many creative works, even if the problems generated by the operating space, have not always been similar.

\subsection{Order - disorder, harmony, composition balance}

The order and disorder of another basic principle in the organization of visual space is a certain way of disposing or arranging the component elements. The manner of distribution within the framework may suggest the idea of logic, organization - when we speak of order - or the idea of spontaneity when we refer to disorder. Representing the main factor in the consolidation of the overall image, the harmony represents a specific relationship, an agreement between the parties and the whole, summing up all the ordering basic components: balance, symmetry, proportion, rhythm. The relationships between the parts, the harmonies, the rhythms, the contrasts are factors of expressivity capable of determining aesthetic emotions and unity in the impression.

It is interesting to note the problem created by placing the different elements within the limits of a surface, considering the visual orientation changes produced, aiming to establish the compositional balance, value of the space. Placing an element in the center of the operating space, static and centripetally balances the respective space, however any derivative of this element towards the sides or corners creates a dynamic tension of imbalance that requires compensation (Arnheim 1995, 225-227). Sometimes, in the case of different elements from a quantitative point of view, the balance can be restored in value or chromatic considering that a smaller element but of a darker value can be harder than a larger element but of a more open value. The balance of the visual surface may also depend on the dates of the center of interest suggested composition, chromatic, value, by the type of aerated - agglomerated relationship or by the distribution of the guidelines etc.

\subsection{The character of the composition}

The character of the compositional space can be static, dynamic or equidistant between static and dynamic, realizing the feeling of floating, instability. Establishing the character of the composition from the beginning requires the formation of clear trajectories of shapes and colors, of hidden paths of orientation. In the case of the dynamic composition, the distribution of the elements, the play of the surfaces, the 
chromatic organization, by the way they are applied, gives the impression that they are launched into space, triggering a movement that seems to have the tendency to extend outside the frame. The frame in this situation no longer constitutes a barrier, no longer closes the form. In this context, the idea underlined by Kandinsky is significant, as the spiritual side of life cannot be included in any particular form. Thus, in the case of the open composition, certain formal lines and routes seem to transgress the predetermined boundaries suggesting spirituality. The attempt to graphically translate the tensions, the inner tension, through the specific language, through the perspective of some meanings, reveals the expressive virtues, the dynamic, active character of the operating space, the expression of movement and energy that it contains.

The way the guidelines are developed can also suggest passive, static surfaces that suggest the idea of tranquility and relaxation. If the guidelines focus the view on an interior area, the component elements being completely substituted for the space, we have in discussion the closed composition.

Analyzing the figurative works of the past, we find that the objects or characters are distributed, organized on the surface according to compositional laws generating rigorous constructive structures. The networks of lines and geometrical forms that structure the works imply the establishment of a system of coordination, of putting in relation in a certain order the elements that enter the composition. This linear framework constitutes the hidden structure of the works having the role of organization, ordering. Whether figurative or non-figurative, the operating space most often has a compositional framework, and reinforcement on which the various elements of visual language constituted in original character substitutions are organized. Our eye tends to establish lines and shapes that can only be understood. The directional lines may attract the look in a certain area of the composition, may induce a certain equilibrium-imbalance, static or dynamic character, etc. Although the overall construction of the painting adds up to theoretical assimilated assumptions over time, yet the true expressiveness, the true "soul" of the painting is not the result of aestheticizing theory and reasoning, but is mainly about sensitivity, enthusiasm for inspiration. It is about intuition and free will in choosing the means and possibilities of combining them because there will never be rules, established premeditated prescriptions that indicate the implementation in a certain way, to produce the expected effect. In such a situation, the spiritual area of creation is activated and, as Kandinsky mentioned, "when the human soul lives stronger, art also becomes more alive, because between soul and art there is a connection, through mutual action and fulfillment". (1994, 111)

Many times we think, we analyze logically, we appeal to reason and everything seems to be easy to achieve, but not the same thing happens when we try to transpose practically the thought. Often just the detachment of any reasoning and the spontaneity appeal leads to the making of valid, expressive configurations, with the strong emotional load. A conclusive example, in this regard, is Jackson Pollock - one of 
the important representatives of the current action - painting, along with Robert Motherwell and Willem de Kooning. The works of this artist release energy, sprung from the gesture - the physical component that involves in the act of creation the whole body of the artist. Preferring large works (some $6 \mathrm{~m}$ long), Pollock simply spreads the paint on the canvas from drilled pots and directs it with a stick. Although the final impression is of spontaneity, rational control is always present during the execution, keeping in balance the tension between the aesthetic image and the conceptual thinking, between the semi-conscious empathy and the awaken consciousness. In making highly refined graphics, he often uses industrial colors, taking into account the specific properties of fluidity, viscosity, leakage, coagulation, drying, etc. (Laneyrie $2002,262)$. The analysis in this direction gives the possibility to keep under control a somewhat unpredictable technique in which the trajectories, mazes, drops and all kinds of leaks overlap, intersecting at an alert pace. The creation, representative of abstract expressionism, is currently crowned with well-deserved appreciation, with numerous retrospectives always being organized in Europe and America.

\subsection{Chromatic harmonies}

The shapes begin to come to life in relation to color tones and we notice that the value of one color can be underlined by one form and subdued by another. Sometimes we can resort to the physical mixing of colors, sometimes to the effect produced by the juxtaposition of pure or broken tones and thus the resulting color is only an optical mixture, a visual impression. The overlap of several layers of color of different tones and different consistencies - from the solid paste to the fluidity of the washings - can also lead to surprising results. The intensity, the vibration, the color ratio in the case of abstract or decorative representations are qualities that exist independently, without suggesting the depth of the visual space induced by the linear perspective. The idea of space, in this situation, is created by the rhythms of colors by means of their own expression, through the thermal perspective, respectively the illusion created by the hot-cold contrast. The warm color tones seem to be positioned in a space closer to the viewer while the cold tones move away. The color ranges can be set exponentially given the affective and intellectual impact of the pictorial image. By allowing manipulation in infinite ways, through their relationships, colors have the ability to suggest a multitude of indications, apart from the purely visual ones. They may suggest sound resonances: acute - pure colors, sudden - broken tones; tactile suggestions: harsh - softness, opacity - transparency, weight; of approach and distance: hot - cold contrast, implicitly thermal differentiation. The adjacent surfaces transmit something to each other, as a reflection of the color of each one, especially in the boundary area between them, is the tendency of the colors to approach as a tone, to equalize (Lazarescu 2009, 195-206). This optical phenomenon can be speculated for the 
unification of the chromatic atmosphere. On the other hand, in other situations, problems with complementary color contrast appear. As we know, the moment the eyes perceive a color it automatically demands compensation through a complementary color. Joining such colors strengthens their power by suggesting a dynamic pulse. The orange, for example, becomes stronger in the presence of blue and will no longer receive from its reflexes as in the above situation. The optical and physical mixture, the reflections that the colors lend to each other are aspects encountered in all stages of work. All these high color issues are required to be welldosed, qualitatively and quantitatively balanced to support the compositional structure, to strengthen the emotion that is desired to be released. Regarding the chromatic composition, Klee talks about the possibility of transforming "errors into virtue" considering that just the deviations from well-established rules can lead to finding new chromatic solutions (Hasan 1999, 423). The artistic activity in general implies the knowledge of the essential laws governing this field but after a good assimilation you already have to go on unexplored lands. This exciting game with the known and the unknown will perpetuate the mind and spirit of the artist.

\subsection{The configuration of artistic structures}

Analyzing the modalities of representation of some structures we notice an impressive range of possibilities. Sometimes the solution is materialized through agglomerations, adjustments, and associations of different techniques, sometimes it becomes obvious the economy of the means and the accuracy of the surface on which simple, precisely elaborated forms are detached painted in shades of the colors laid flat or superimposed through the transparency of the pigments. Other times the structure becomes paramount with optical-spatial and expressive properties. The combination of elaborate geometric signs or forms, having their own composition status, can lead to the formation of modules which in turn are repeated, following different directions, in order to create a new complex configuration that ensures the harmonious ordering of a surface. The relationships between the parts, the color harmonies, the rhythms and the value contrasts, are factors of the plastic expressiveness capable of determining aesthetic emotions. The relationship between essence and appearance, between the structural and the individual aims to order the heterogeneous parts of the represented elements. They become homogeneous microstructures of visual language, to produce on the viewer emotion and unity in the impression. The shapes taken from everyday reality can be interpreted until they become elements of a composition with purely plastic valences. The world around us is made up of shapes and colors, which never cease to amaze and arouse interest in discovering and exploiting them. Each object has a certain form, in a certain way composed, in a certain way structured, with characteristics worthy of study. Observing them gives us the possibility to understand 
that each element, object or phenomenon has its own structure, an order in its configuration, development and evolution. This order can also be transposed by the act of artistic creation in order to obtain a higher expressivity, a special effect.

\section{Conclusions}

The liberation of the imagination in the configuration of an original space in which the affection and the thought work together harmoniously generates aesthetic accomplishments. All these things used and exploited by the artist and, implicitly, transmitted to others lead to the outlining of moral and spiritual values, to the completion of human evolution. A work of art once configured generates aesthetic and spiritual fulfillments, but at the same time new challenges, new inner disturbances are born. The analysis and interpretation of the artistic phenomenon in relation to other cultures plays an essential role in consolidating distinct, personalized conceptions, but keeping in line with current requirements.

\section{References}

Ailincăi, Cornel. 1982. Introduction to the Grammar of Visual Language. ClujNapoca: Dacia Publishing House.

Argan, Giulio Carlo. 1982. Modern Art. Bucharest: Meridiane Publishing House.

Arnheim, Rudolf. 1995. The Force of the Visual Center. Bucharest: Meridiane Publishing House.

Gadamer, Hans-Georg. 2000. The Relevance of the Beautiful. Bucharest: Polirom Publishing House.

Hasan, Yvonne. 1999. Paul Klee and Modern Painting. Bucharest: Meridiane Publishing House.

Huyghe, Rene. 1981. Dialogue with the Visible. Bucharest: Meridiane Publishing House.

Kandinsky, Wassily. 1994. The Spiritual in Art. Bucharest: Meridiane Publishing House.

Laneyrie Dagen, Nadeije. 2002. Painting-Secrets and Disclosures. Bucharest: RAO Publishing House.

Lăzărescu, Liviu. 2009. The Color in Art. Bucharest: Polirom Publishing House.

Ortega y Gasset, José. 2000. The Dehumanization of Art. Bucharest: Humanitas Publishing House. 